PROCEEDINGS OF THE

AMERICAN MATHEMATICAL SOCIETY

Volume 127, Number 11, Pages 3383-3391

S 0002-9939(99)04866-2

Article electronically published on May 3, 1999

\title{
ON THE DARBOUX THEOREM FOR WEAK SYMPLECTIC MANIFOLDS
}

\author{
DARIO BAMBUSI
}

(Communicated by Peter Li)

\begin{abstract}
A new tool to study reducibility of a weak symplectic form to a constant one is introduced and used to prove a version of the Darboux theorem more general than previous ones. More precisely, at each point of the considered manifold a Banach space is associated to the symplectic form (dual of the phase space with respect to the symplectic form), and it is shown that the Darboux theorem holds if such a space is locally constant. The following application is given. Consider a weak symplectic manifold $M$ on which the Darboux theorem is assumed to hold (e.g. a symplectic vector space). It is proved that the Darboux theorem holds also for any finite codimension symplectic submanifolds of $M$, and for symplectic manifolds obtained from $M$ by the Marsden-Weinstein reduction procedure.
\end{abstract}

\section{INTRODUCTION}

It was first pointed out by Marsden [1] that the Darboux theorem fails for weak symplectic forms. Afterwards, some generalizations of the theorem to weak symplectic manifolds were proved [2] (see also [3]), but a complete characterization of the situations in which the symplectic form can be locally reduced to a constant one is still lacking. In the present paper a new tool to study reducibility of a weak symplectic form to a constant one is introduced and used to prove a version of the Darboux theorem more general than previous ones. A necessary condition for the Darboux theorem is also obtained.

The main idea of this paper is (following [4]) to associate, at each point of the manifold, a suitable Banach space to the symplectic form (classifying space); it turns out that the Darboux theorem can be proved if the classifying space does not depend on the point of the manifold and a suitable smoothness condition holds. Applying this version of the Darboux theorem the following results are proven: Let $M$ be a weak symplectic manifold in which the Darboux theorem is assumed to hold (e.g. a symplectic vector space); then the Darboux theorem holds for each closed symplectic submanifold of $M$ having finite codimension (see Theorem 3.1). Then, the case where a finite dimensional Lie group acts symplectically on $M$ is considered: it is proven that the Darboux theorem holds for the symplectic manifold obtained

Received by the editors December 1, 1997 and, in revised form, January 21, 1998.

1991 Mathematics Subject Classification. Primary 58B20; Secondary 58F05.

This work was supported by grants CE n. CHRX-CT93-0330/DG, "Order and chaos in conservative dynamical systems", and CE. n. ERBCHRXCT940460 "Stability and universality in classical mechanics". 
from $M$ by the Marsden-Weinstein reduction procedure (see Corollary 3.5). As far as I know these results are new.

I come to the necessary condition for the reducibility of a weak symplectic form to a constant one. It turns out that the assumptions of the present version of the Darboux theorem are not invariant under general coordinate transformations, and so they do not directly provide a necessary condition for the reducibility of the symplectic form to a constant one. Anyway it is possible to exploit the classifying space to give a completely coordinate independent necessary condition for the reducibility of a symplectic form to a constant one. Such a condition is exploited to give a counterexample to the Darboux theorem. Actually this is just a variant of Marsden's counterexample, and is given here mainly for the sake of completeness.

\section{A NECESSARY AND A SUfFicient CONDITION FOR the DARBoux THEOREM}

2.1 Statements. First, recall that a bilinear form $\Omega: \mathcal{P} \times \mathcal{P} \rightarrow \mathbb{R}, \mathcal{P}$ being a Banach space, is said to be weakly nondegenerate if

$$
\{\Omega(X, Y)=0, \quad \forall Y \in \mathcal{P}\} \Longrightarrow X=0 .
$$

It is well known that $\Omega$ defines a linear map $\Omega^{b}: \mathcal{P} \rightarrow \mathcal{P}^{*}$ by

$$
\left\langle\Omega^{b} Y, X\right\rangle:=\Omega(Y, X),
$$

and that, by (1), such a map is injective; if it is also surjective, then $\Omega$ is said to be strongly nondegenerate.

A weak symplectic manifold $(M, \Omega)$ is a differentiable manifold $M$ endowed with a closed differentiable 2 -form $\Omega$, which at each $x \in M$ is weakly nondegenerate as a bilinear form on $T_{x} M$. I will denote by $\Omega_{x}: \mathcal{P} \times \mathcal{P} \rightarrow \mathbb{R}$ the value of $\Omega$ at $x \in M$, and by $J_{x}$ the inverse of the map $\Omega_{x}^{b}$. $J_{x}$ is usually called the Poisson tensor.

Since the Darboux theorem is a local result, it is enough to consider the case where $M$ is an open set $\mathcal{U}$ of a Banach space $\mathcal{P}$ which will be assumed to be reflexive; I will assume also $0 \in \mathcal{U}$, and all functions will be assumed to be $C^{\infty}$.

Using $\Omega_{x}$ define on $\mathcal{P}$ the norm

$$
\|X\|_{\mathcal{F}_{x}}:=\sup _{\|Y\|_{\mathcal{P}}=1}\left|\Omega_{x}(X, Y)\right|=\left\|\Omega_{x}^{b} X\right\|_{\mathcal{P}^{*}},
$$

and consider the completion $\mathcal{F}_{x}$ of $\mathcal{P}$ in such a norm.

Definition. The space $\mathcal{F}_{x}$ thus obtained will be called "the dual of $\mathcal{P}$ with respect to $\Omega_{x}$."

It is clear that $\Omega_{x}$ can be extended to a continuous bilinear form on $\mathcal{P} \times \mathcal{F}_{x}$.

Theorem 2.1. Assume that there exists a neighbourhood $\mathcal{W}$ of 0 such that, for all $x \in \mathcal{W}$ the spaces $\mathcal{F}_{x}$ coincide (i.e. the norms $\|\cdot\|_{\mathcal{F}_{x}}$ are equivalent), and moreover that the map $x \mapsto \Omega_{x}$ is differentiable as an application from $\mathcal{W}$ to the continuous bilinear forms on $\mathcal{F} \times \mathcal{P}$, where $\mathcal{F}:=\mathcal{F}_{x}$; then there exist a neighbourhood $\mathcal{V}$ of 0 and a change of coordinates $\psi$ defined on $\mathcal{V}$ which reduces $\Omega$ to the constant two form $\Omega_{0}:=\left.\Omega_{x}\right|_{x=0}$, namely such that

$$
\psi^{*} \Omega=\Omega_{0} .
$$

Remark 2.2. One can conjecture that the differentiability of $\Omega$ as an application form $\mathcal{V}$ to the bilinear forms on $\mathcal{P} \times \mathcal{F}$ is automatic. It is easy to construct a 
counterexample in the case of $\Omega_{x}$ which is $C^{k}(k<\infty)$ dependent on $x$, however I do not have a counterexample in the $C^{\infty}$ case.

Remark 2.3. The Darboux theorem proved in [3] is contained in the above theorem 2.1. This is easily seen exploiting the lemma on p.10 of [3]. The same holds for the Darboux theorem proved in [2], which is based on ideas quite close to those presented here.

Theorem 2.1 can be reformulated in terms of the Poisson tensor. Indeed the norm $\|X\|_{\mathcal{F}_{x}}$ can obviously be defined by $\|X\|_{\mathcal{F}_{x}}:=\left\|J_{x}^{-1} X\right\|_{\mathcal{P}^{*}}$, and this allows us to obtain the following

Corollary 2.4. Define $\mathcal{F}$ as the completion of $\mathcal{P}$ in the norm $\|X\|_{\mathcal{F}}:=\left\|J_{0}^{-1} X\right\|_{\mathcal{P}^{*}}$, where $J_{0}:=\left.J_{x}\right|_{x=0}$. Assume that there exists a neighbourhood of the origin where $J_{x}$ extends to a bounded linear operator from $\mathcal{P}^{*}$ to $\mathcal{F}$ smoothly dependent on $x$; then there exists a chart about zero in which the symplectic form (and therefore the Poisson tensor) is constant.

Remark 2.5. The constancy of the spaces $\mathcal{F}_{x}$ is a property which is not completely chart invariant; indeed one can construct examples of change of coordinates which change the spaces $\mathcal{F}_{x}$ everywhere, but not at one single point.

One can use the spaces $\mathcal{F}_{x}$ to give a coordinate independent necessary condition for the Darboux theorem.

Proposition 2.6. The existence of chart about 0 in which the symplectic form is constant implies that there exists a neighbourhood of 0 such that for each $x$ in such a neighbourhood there exists an isomorphism between $\mathcal{F}_{x}$ and $\mathcal{F}_{0}$ which restricts to an isomorphism of $\mathcal{P}$ with itself.

2.2. Proofs. The proof of Theorem 2.1 will be obtained along the lines of [5] (which follows an idea by Moser). The original part consists of the proof of the smoothness of the vector field generating the transformation we are looking for.

We begin with two simple lemmas. Consider the map $\tilde{\Omega}_{x}^{b}: \mathcal{F}_{x} \rightarrow \mathcal{P}^{*}$ defined by $\left\langle\tilde{\Omega}_{x}^{b} V ; W\right\rangle:=\Omega_{x}(V, W)$; we have the following

Lemma 2.7. $\tilde{\omega}_{x}^{b}$ is an isomorphism between $\mathcal{F}_{x}$ and $\mathcal{P}^{*}$.

Proof. It is immediate to remark that $\tilde{\Omega}_{x}^{b}$ is a linear unitary operator, so that it is invertible on its range, and its inverse is continuous. I prove now that it is surjective. Denote by $\mathcal{R}$ the range of $\tilde{\Omega}_{x}^{b}$ (which is close by the above consideration) and assume by contradiction that there exists a covector $\alpha \in \mathcal{P}^{*}$ such that $\alpha \notin \mathcal{R}$. By the Hahn-Banach theorem there exists a $X \in \mathcal{P}^{* *}$ such that

$$
X(\alpha)=1
$$

and

$$
X(\beta)=0, \quad \forall \beta \in \mathcal{R} .
$$

But $\mathcal{P}$ is reflexive, and therefore $X \in \mathcal{P}$, so (5) is equivalent to

$$
\Omega_{x}(Y, X)=0, \forall Y \in \mathcal{F}_{x},
$$

which, by (1), implies $X=0$ against (4). 
The above lemma is used to prove

Lemma 2.8. The map $\Omega_{x}^{b}: \mathcal{P} \rightarrow \mathcal{F}_{x}^{*}$, defined by $X \mapsto \Omega_{x}(X,$.$) , is an isomorphism.$

Proof. It is clear that the map is injective; I prove now that it is surjective by constructing its inverse. Consider the map that associates to any $\alpha \in \mathcal{F}_{x}^{*}$ the vector $X \in \mathcal{P}^{* *}$ defined by

$$
X(\beta):=-\alpha\left(J_{x} \beta\right), \forall \beta \in \mathcal{P}^{*},
$$

where $J_{x}:=\left(\tilde{\Omega}_{x}^{b}\right)^{-1}$. But $\mathcal{P}$ is reflexive, so that $X \in \mathcal{P}$. It is easy to see that this map is the inverse of $\Omega_{x}^{b}$.

In what follows we will not distinguish between $\Omega_{x}^{b}$ and $\tilde{\Omega}_{x}^{b}$.

Remark 2.9. Lemmas 2.7 and 2.8 hold also for any nondegenerate bilinear form (skew-symmetry plays no role).

Remark 2.10. In the hypotheses of Theorem 2.1 the map

$$
\begin{aligned}
\mathcal{W} & \rightarrow L\left(\mathcal{P}, \mathcal{F}^{*}\right), \\
x & \mapsto \Omega_{x}^{b}
\end{aligned}
$$

is smooth; here $L\left(\mathcal{P}, \mathcal{F}^{*}\right)$ is the Banach space of linear operators from $\mathcal{P}$ to $\mathcal{F}^{*}$. Indeed it is the composition of the map $x \mapsto \Omega_{x}$ which is smooth as an application from $\mathcal{W}$ to the bilinear forms on $\mathcal{P} \times \mathcal{F}$ by hypothesis, and the linear map ${ }^{b}: \Omega \mapsto \Omega^{b}$, which is analytic.

Proof of Theorem 2.1. Denote $\bar{\Omega}:=\Omega_{0}-\Omega$ and $\Omega^{t}:=\Omega+t \bar{\Omega}$, where $\Omega_{0}:=\left.\Omega_{x}\right|_{x=0}$. By the Poincaré lemma we have

$$
\bar{\Omega}=d \alpha, \quad \text { with } \alpha_{x} X:=\int_{0}^{1} s \bar{\Omega}_{s x}(x, X) d s .
$$

We look for a smooth vector field $Y_{t}: \mathcal{P} \rightarrow \mathcal{P}$ such that

$$
i_{Y_{t}} \Omega^{t}=-\alpha
$$

By (7) the one form $\alpha_{x}$ can be extended to an element of $\mathcal{F}^{*}$. Consider now

$$
\left(\Omega^{t}\right)_{x}^{b}: \mathcal{P} \rightarrow \mathcal{F}^{*}
$$

When $x=0$, one has $\left.\Omega_{x}^{t}\right|_{x=0} \equiv \Omega_{0}$, and therefore at such point (9) is an isomorphism. By Remark $2.10\left(\Omega_{x}^{t}\right)^{b}$ depends smoothly on $x$ and, for $x$ close to zero, it is an isomorphism. So, one can define

$$
Y_{t}:=\left(\left(\Omega_{x}^{t}\right)^{b}\right)^{-1} \alpha
$$

and $Y_{t}$ is a smooth, time dependent vector field taking values in $\mathcal{P}$.

Then the proof is easily completed following the scheme by Moser. So, let $F_{t}$ be the flow generated by $Y_{t}$; then

$$
\frac{d}{d t} F_{t}^{*} \Omega_{t}=F_{t}^{*}\left(£_{Y_{t}} \Omega_{t}\right)+F_{t}^{*} \frac{d}{d t} \Omega_{t}=F_{t}^{*}(-d \alpha+\bar{\Omega})=0,
$$

so $F_{1}^{*} \Omega_{1}=\Omega$, and $F_{1}^{-1}$ is the desired change of variables. 
Proof of Corollary 2.4. By Lemma 2.7, $J_{0}$ is an isomorphism of $\mathcal{P}^{*}$ and $\mathcal{F}$, it follows (from the implicit function theorem) that in a neighbourhood of zero also $J_{x}$ is an isomorphism of $\mathcal{P}^{*}$ and $\mathcal{F}$, and therefore the spaces $\mathcal{F}_{x}$ coincide with $\mathcal{F}$; moreover one clearly has that

$$
\begin{aligned}
\Omega_{x}: \mathcal{F} \times \mathcal{P} & \rightarrow \mathbb{R} \\
(X, Y) & \mapsto\left\langle J_{x}^{-1} X, Y\right\rangle
\end{aligned}
$$

depends smoothly on $x$ as a bilinear form on $\mathcal{F} \times \mathcal{P}$, therefore Theorem 2.1 gives the result.

Proof of Proposition 2.6. Assume that there exists a change of coordinates $\psi$ about 0 such that $\psi^{*} \Omega=\Omega_{0}$. Then, for $X, Y \in \mathcal{P}$ one has

$$
\Omega_{x}\left(\psi_{* x} X, \psi_{* x} Y\right)=\Omega_{0}(X, Y)
$$

where we denoted by $\psi_{* x}$ the tangent map to $\psi$ at $x$. Denote by $A_{x}$ the inverse of $\psi_{* x}$; then one has

$$
\Omega_{x}(X, Y)=\Omega_{0}\left(A_{x} X, A_{x} Y\right)
$$

and therefore

$$
\left\|A_{x} Y\right\|_{\mathcal{F}_{0}}=\sup _{Z \neq 0} \frac{\left|\Omega_{0}\left(Z, A_{x} Y\right)\right|}{\|Z\|_{\mathcal{P}}}=\sup _{X \neq 0} \frac{\left|\Omega_{0}\left(A_{x} X, A_{x} Y\right)\right|}{\left\|A_{x} X\right\|_{\mathcal{P}}}=\sup _{X \neq 0} \frac{\left|\Omega_{x}(X, Y)\right|}{\left\|A_{x} X\right\|_{\mathcal{P}}}
$$

where $X=A_{x}^{-1} Z$. But, since $A_{x}$ is an isomorphism, one has

$$
\left\|A_{x} X\right\|_{\mathcal{P}} \geq\left\|A_{x}^{-1}\right\|^{-1}\|X\|_{\mathcal{P}},
$$

from which, substituting in (10) and taking into account the definition of the norm $\mathcal{F}_{x}$

$$
\left\|A_{x} Y\right\|_{\mathcal{F}_{0}} \leq\left\|A_{x}^{-1}\right\|\|Y\|_{\mathcal{F}_{x}}
$$

It follows that $A_{x}$ can be extended to a continuous operator from $\mathcal{F}_{x}$ to $\mathcal{F}_{0}$; moreover, the same holds for the operator $A_{x}^{-1}$, so $A_{x}$ is the desired isomorphism.

\section{Applications to Symplectic SUbmanifolds}

Let $M$ be a weakly symplectic manifold modelled on a reflexive Banach space; assume that at each point of $M$ there exists a chart in which the symplectic form is constant. The following theorem will be proved.

Theorem 3.1. Let $N \subset M$ be a closed symplectic submanifold of $M$ having finite codimension. Then at each point of $N$ there exists a chart in which the symplectic form of $N$ is constant.

Before giving the proof of Theorem 3.1 I give two lemmas on symplectic vector spaces which will be used below.

Let $(\mathcal{P}, \Omega)$ be a weakly symplectic vector space, with $\mathcal{P}$ a reflexive Banach space; as above denote by $\mathcal{F}$ the dual of $\mathcal{P}$ with respect to $\Omega$. Given a closed subspace $V \subset \mathcal{P}$ define $\bar{V}^{\mathcal{F}}$ to be the closure of $V$ in the topology of $\mathcal{F}$, and

$$
\begin{aligned}
V^{\perp} & :=\{X \in \mathcal{P}: \Omega(X, Y)=0 \forall Y \in V\}, \\
V^{\dagger} & :=\{X \in \mathcal{F}: \Omega(X, Y)=0 \forall Y \in V\} .
\end{aligned}
$$


Note that

$$
{\overline{V^{\perp}}}^{\mathcal{F}}=V^{\dagger} .
$$

If $W \subset \mathcal{F}$ is a closed subspace, I will denote

$$
W^{\perp}:=\{X \in \mathcal{P}: \Omega(X, Y)=0 \forall Y \in W\} ;
$$

this is not ambiguous since (for $V \subset \mathcal{P}$ ) one has

$$
V^{\perp}=\left(\bar{V}^{\mathcal{F}}\right)^{\perp} .
$$

Lemma 3.2. Let $V \subset \mathcal{P}$ be a closed subspace; then one has

$$
V^{\perp \perp}=V, \quad\left(V^{\perp}\right)^{\dagger}=\bar{V}^{\mathcal{F}} .
$$

Proof. Let $X \notin V$ be a vector of $\mathcal{P}$; then by the Hahn-Banach theorem there exists $\alpha \in \mathcal{P}^{*}$ such that $\alpha(X)=1$ and $\left.\alpha\right|_{V}=0$. Consider $J \alpha \in \mathcal{F}$ : clearly one has $J \alpha \in V^{\dagger}$ and $\Omega(J \alpha, X)=1$, so that

$$
X \notin\left(V^{\dagger}\right)^{\perp}=V^{\perp \perp}
$$

where the last equality follows from (11) and (12). This proves $X \in V \Longrightarrow X \in$ $V^{\perp \perp}$; the inverse inclusion is trivial. To obtain the second of (13) remark that

$$
\bar{V}^{\mathcal{F}}={\overline{V^{\perp \perp}}}^{\mathcal{F}}=\left(V^{\perp}\right)^{\dagger},
$$

where the second equality follows from (11).

For a previous proof of $V^{\perp \perp}=V$ in weak symplectic spaces see [6].

Lemma 3.3. Let $V \subset \mathcal{P}$ be closed and symplectic; then

1 .

$$
\mathcal{P}=V \oplus V^{\perp} .
$$

2. Let $\pi$ be the projection on $V$; then $\pi$ extends to a continuous map from $\mathcal{F}$ to $\bar{V}^{\mathcal{F}}$.

3. There exists $C$ such that $\forall X \in V$ one has

$$
\|X\|_{\mathcal{F}} \leq C \sup _{Y \in V \backslash\{0\}} \frac{|\Omega(X, Y)|}{\|Y\|} .
$$

Proof. Clearly one has $V \cap V^{\perp}=\{0\}$ and $\bar{V}^{\mathcal{F}} \cap V^{\dagger}=\{0\}$. Define $\mathcal{P}_{1}:=V \oplus V^{\perp}$, and assume that it does not coincide with $\mathcal{P}$; then there exists $0 \neq X \in \mathcal{P} \backslash \mathcal{P}_{1}$. By the Hahn-Banach theorem there exists $\alpha \in \mathcal{P}^{*}$ such that $\alpha(X)=1$ and $\left.\alpha\right|_{\mathcal{P}_{1}}=0$. Then, by Lemma 3.2, one has

$$
J \alpha \in V^{\dagger} \cap\left(V^{\perp}\right)^{\dagger}=V^{\dagger} \cap \bar{V}^{\mathcal{F}}=\{0\} .
$$

Since $\alpha \neq 0$, this is a contradiction. To prove 2 fix $X \in \mathcal{P}$; we have

$$
\begin{gathered}
\|\pi X\|_{\mathcal{F}}=\sup _{Y \in \mathcal{P} \backslash\{0\}} \frac{|\Omega(\pi X, Y)|}{\|Y\|}=\sup _{Y \in \mathcal{P} \backslash\{0\}} \frac{|\Omega(\pi X, \pi Y)|}{\|Y\|} \\
=\sup _{Y \in \mathcal{P} \backslash\{0\}} \frac{|\Omega(X, \pi Y)|}{\|Y\|} \leq \sup _{Y \in \mathcal{P} \backslash\{0\}} \frac{\|\pi Y\|\|X\|_{\mathcal{F}}}{\|Y\|} \leq C\|X\|_{\mathcal{F}},
\end{gathered}
$$


where the last equality is just boundedness of $\pi$. I come to 3. Fix $Y \in V$; we have

$$
\begin{aligned}
\|Y\|_{\mathcal{F}}=\sup _{X \in \mathcal{P} \backslash\{0\}} \frac{|\Omega(X, Y)|}{\|X\|} & =\sup _{X \in \mathcal{P} \backslash\{0\}} \frac{|\Omega(\pi X, Y)|}{\|X\|} \\
& \leq \sup _{X \in \mathcal{P} \backslash\{0\}} \frac{|\Omega(\pi X, Y)|}{\frac{\|\pi X\|}{C}}
\end{aligned}
$$

where the last equality is due to the boundedness of $\pi$. From this 3 immediately follows.

Proof of Theorem 3.1. The result is local, so I consider only the case where $M=$ $\mathcal{U} \subset \mathcal{P}$ is an open subset of $\mathcal{P}$ endowed by a constant symplectic form $\Omega$. Moreover assume $0 \in \mathcal{U} \cap N$; I will construct a chart of $N$ about 0 in which the assumptions of Theorem 2.1 are satisfied. Denote by $\mathcal{F}$ the dual of $\mathcal{P}$ with respect to $\Omega$, and $V:=T_{0} N$; then $V$ is symplectic and therefore $\mathcal{P}=V \oplus V^{\perp}$; denote by $\pi: \mathcal{P} \supset$ $\mathcal{U} \rightarrow V$ the projection on $V$. Clearly there exists a neighbourhood $\mathcal{W} \subset N$ of 0 where

$$
\begin{aligned}
\varphi: \mathcal{W} & \rightarrow V \\
x & \mapsto \varphi(x):=\pi x
\end{aligned}
$$

is a chart of $N$. I claim this is a chart in which the assumptions of Theorem 2.1 are satisfied.

To start with note that in this chart the expression $\Omega^{N}$ of the symplectic form of $N$ is given by

$$
\begin{aligned}
\Omega_{x}^{N}: V \times V & \rightarrow \mathbb{R} \\
(X, Y) & \mapsto \Omega_{x}^{N}(X, Y):=\Omega\left(\varphi_{* x}^{-1} X, \varphi_{* x}^{-1} Y\right) ;
\end{aligned}
$$

I will show that $\varphi_{* x}$ extends to an isomorphism of ${\overline{T_{x} N}}^{\mathcal{F}}$ and $\bar{V}^{\mathcal{F}}$; then the result will easily follow.

First note that, from the linearity of $\pi$, one has

$$
\begin{aligned}
\varphi_{* x}: T_{x} N & \rightarrow V \\
X & \mapsto \varphi_{* x} X=\pi X .
\end{aligned}
$$

I use this fact to construct more explicitly $\varphi_{* x}^{-1}$. Fix a basis $v_{1}(x), \ldots, v_{2 n}(x)$ of $\left(T_{x} N\right)^{\perp}$ smoothly dependent on $x$, define the one forms $\alpha_{i}^{x}:=\Omega\left(v_{i}(x),.\right)$, and note that they extend to elements of $\mathcal{F}^{*}$ smoothly dependent on $x$. Then define the linear operator

$$
\begin{aligned}
A_{x}: \mathcal{P} & \rightarrow V \oplus \mathbb{R}^{2 n} \\
X & \mapsto\left(\pi X ; \alpha_{1}^{x} X, \ldots, \alpha_{2 n}^{x} X\right) ;
\end{aligned}
$$

clearly $\left.A_{x}\right|_{T_{x} N} \equiv\left(\varphi_{* x}, 0\right)$. It is immediate to realize that $A_{0}$ is an isomorphism which extends to an isomorphism of $\mathcal{F}$ with $\bar{V}^{\mathcal{F}} \oplus \mathbb{R}^{2 n}$. Moreover, $A_{x}$ depends smoothly on $x$ both as linear bounded operator from $\mathcal{P}$ to $V \oplus \mathbb{R}^{2 n}$ and as bounded linear operator from $\mathcal{F}$ to $\bar{V}^{\mathcal{F}} \oplus \mathbb{R}^{2 n}$. So there exists a neighbourhood $\mathcal{W}$ of $0 \subset \mathcal{U}$ where it is an isomorphism. It follows that

$$
\begin{aligned}
\varphi_{* x}^{-1} & : V \rightarrow T_{x} N \\
X & \mapsto A_{x}^{-1}(X, 0)
\end{aligned}
$$

extends to an isomorphism of $\bar{V}^{\mathcal{F}}$ with ${\overline{T_{x} N}}^{\mathcal{F}}$ smoothly dependent on $x$. 
Denote now by $F_{x}$ the dual of $V$ with respect to $\Omega_{x}^{N}$. By Lemma 3.3 (assertion 3), one has $F_{0}=\bar{V}^{\mathcal{F}}$. I am going to prove that the same holds for $x$ in a neighbourhood of 0 . By the very definition of $\|\cdot\|_{F_{x}}$ and of $\Omega_{x}^{N}$, for $Y \in V$ one has

$$
\|Y\|_{F_{x}}=\sup _{X \in T_{x} N \backslash\{0\}} \frac{\left|\Omega\left(X, \varphi_{* x}^{-1} Y\right)\right|}{\left\|\varphi_{* x} X\right\|},
$$

but, since $\varphi_{* x}$ is an isomorphism of $T_{x} N$ and $V$ there exist constants such that

$$
\begin{gathered}
\|Y\|_{F_{x}} \leq C_{1}\left\|\varphi_{* x}^{-1} Y\right\|_{\mathcal{F}} \leq C_{2}\|Y\|_{\mathcal{F}} \\
\|Y\|_{F_{x}} \geq C_{3} \sup _{X \in T_{x} N \backslash\{0\}} \frac{\left|\Omega\left(X, \varphi_{* x}^{-1} Y\right)\right|}{\|X\|} \geq C_{4}\left\|\varphi_{* x}^{-1} Y\right\|_{\mathcal{F}} \geq C_{5}\|Y\|_{\mathcal{F}},
\end{gathered}
$$

where I used also the fact that $\varphi_{* x}$ is an isomorphism from $\bar{V}^{\mathcal{F}}$ and $\bar{T}_{x} N^{\mathcal{F}}$, and Lemma 3.3.

Finally one has to prove that $\Omega_{x}^{N}$ depends smoothly on $x$ as a bilinear form on $V \times F \simeq V \times \bar{V}^{\mathcal{F}}$. But this is a trivial consequence of the smoothness of $A_{x}^{-1}$ both as a map from $V \oplus \mathbb{R}^{2 n}$ to $\mathcal{P}$ and as a map from $\bar{V}^{\mathcal{F}} \oplus \mathbb{R}^{2 n}$ to $\mathcal{F}$, and of the definition of $\Omega^{N}$.

Remark 3.4. I think that Theorem 3.1 should hold also for $\infty$-codimensional symplectic submanifolds of $M$; however, I was not able to prove that $\left(T_{x} N\right)^{\perp}$ depends smoothly on $x$, and therefore I did not succeed in defining an operator of the kind of the operator (14)

I come to symplectic manifolds obtained by the Marsden-Weinstein reduction procedure.

Fix again $M$ as above, and let $G$ be a finite dimensional Lie group. Let $\Phi$ : $M \times G \rightarrow M$ be a smooth symplectic action of $G$ on $M$ admitting an $A d^{*}$-equivariant momentum map $I: M \rightarrow g^{*}$. Let $\mu \in g^{*}$ be a regular value of $I$, and denote by $G_{\mu}$ the isotropy group of $\mu$, namely

$$
G_{\mu}:=\left\{g \in G: A d_{g}^{*} \mu=\mu\right\} .
$$

Assume $G_{\mu}$ acts properly and freely on $I^{-1}(\mu)$, and denote

$$
M_{\mu}:=\frac{I^{-1}(\mu)}{G_{\mu}}
$$

then it is well known that $M_{\mu}$ is a symplectic manifold.

Corollary 3.5. About each point of $M_{\mu}$ there exists a chart in which the symplectic form is constant.

Proof. Let $z \in M_{\mu}$. Then $z=[x]$ for some $x \in I^{-1}(\mu)$; denote by $G_{\mu} x$ the orbit of $G_{\mu}$ through $x$. Let $P \subset M$ be a closed submanifold of $M$ passing through $x$ such that $T_{x} M \equiv T_{x} P \oplus T_{x}\left(G_{\mu} x\right)$. Then it is well known that $M_{\mu}$ is locally isomorphic, as a symplectic manifold, to a neighbourhood of $x$ in $P \cap I^{-1}(\mu)$. But $P \cap I^{-1}(\mu)$ is a finite codimension submanifold of $M$, and therefore Theorem 3.1 gives the result. 


\section{A counterexample to the Darboux theorem (following Marsden)}

Let $Q$ be a Banach manifold modelled on a reflexive Banach space $\mathcal{B}$; then it is well known that the canonical symplectic structure of $T^{*} Q$ is strong [1]. Suppose that $Q$ is endowed by a metric; then it is possible to pull back the symplectic form of $T^{*} Q$ to $T Q$. If the metric of $Q$ is only weakly nondegenerate (i.e. $g_{x}: T_{x} Q \times T_{x} Q \rightarrow$ $\mathbb{R}$ is weakly nondegenerate as a bilinear form), then the symplectic form on $T Q$ is only weakly nondegenerate. Marsden's idea is to define a symplectic form $\Omega$ on $T Q$ using a metric, which for a given $x_{0} \in Q$ is only weakly nondegenerate, while it is strongly nondegenerate at the points $x_{n}$ of a sequence having $x_{0}$ as an accumulation point. It clearly turns out that $\forall X \in T_{x_{0}} Q$ the form $\Omega_{X}$ is weakly nondegenerate, and therefore $\mathcal{F}_{X}$ strictly contains $T_{X} T Q \simeq \mathcal{B} \times \mathcal{B}$, while for any $Y \in T_{x_{n}} Q$ the form $\Omega_{Y}$ is strongly nondegenerate, and therefore $\mathcal{F}_{Y} \equiv T_{Y} T Q \simeq \mathcal{B} \times \mathcal{B}$. This violates the necessary condition 2.6, and therefore the Darboux theorem fails.

A concrete example of this kind can be obtained choosing $Q$ to be the Sobolev space $H^{1}\left(\mathbf{T}^{1}\right)$ of $L^{2}$ functions on $[0, \pi]$ having $L^{2}$ weak derivatives, and fulfilling periodic boundary conditions; the weak metric can be defined by

$$
g_{u}(X, Y):=\int_{\mathbf{T}^{1}} X(x) Y(x) d x+\|u\|_{L^{2}}^{2} \int_{\mathbf{T}^{1}} X_{x}(x) Y_{x}(x) d x,
$$

which at $u=0$ is weak (it is just the $L^{2}$ pairing) while for $u \neq 0$ it is strong.

A simple calculation shows that, denoting (as usual) by $H^{-1}$ the dual of $H^{1}\left(\mathbf{T}^{1}\right)$ with respect to the $L^{2}$ pairing, the map

$$
\Omega_{(u, \dot{u})}^{b}: H^{1}\left(\mathbf{T}^{1}\right) \times H^{1}\left(\mathbf{T}^{1}\right) \rightarrow H^{-1} \times H^{-1}
$$

corresponding to the so obtained symplectic form is given by

$$
\Omega_{(u, \dot{u})}^{b}\left(\begin{array}{c}
V \\
\dot{V}
\end{array}\right)=\left(\begin{array}{c}
-\|u\|_{L^{2}}^{2} \dot{V}_{x x}+\dot{V}-\dot{u}_{x x} 2\langle u, V\rangle_{L^{2}}+2\left\langle V, \dot{u}_{x x}\right\rangle u \\
\|u\|_{L^{2}}^{2} V_{x x}-V
\end{array}\right)
$$

which is an isomorphism for $u \neq 0$.

Remark 4.1. Choosing $Q=H^{s}\left(\mathbf{T}^{1}\right)(s>1)$ endowed by the metric (15) one obtains a symplectic form which is nowhere strong, however exploiting (16) it is easy to show that also in this case the Darboux theorem fails. Indeed it is clear that the reason why the Darboux theorem is violated is that such a map is a differential operator whose degree changes at $u=0$.

\section{REFERENCES}

[1] J. E. Marsden: Darboux's Theorem Fails for Weak Symplectic Forms. Proc. Amer. Math. Soc. 32, 590-592 (1972). MR 45:2755

[2] A.J. Tromba: Almost Riemannian Structures on Banach Manifolds: the Morse Lemma and the Darboux Theorem. Canad. J. Math. 28, 640-652 (1976). MR 53:6613

[3] J.E. Marsden: Lectures on Geometric Methods in Mathematical Physics. SIAM (Philadelphia, 1981). MR 82j:58046

[4] D. Bambusi, A. Giorgilli: Exponential Stability of States Close to Resonance in Infinite Dimensional Hamiltonian Systems. Jour. Stat. Phys. 71, p. 569-606 (1993). MR 94m:58198

[5] A. Weinstein: Symplectic Structures on Banach Manifolds. Bull. Amer. Math. Soc. 75, 10401041 (1969)

[6] R. Schmid: Infinite dimensional Hamiltonian systems. Bibliopolis (Napoli 1987). MR 90f: 58072

Dipartimento di Matematica dell’Università, Via Saldini 50, 20133 Milano, Italy

E-mail address: bambusi@mat.unimi.it 